\title{
IMPLICAÇÕES DO ALCOOLISMO E DA DOENÇA HEPÁTICA CRÔNICA SOBRE O METABOLISMO DE MICRONUTRIENTES ${ }^{+}$
}

\author{
Regiane MAIO*, Jane Bandeira DICHI** e Roberto Carlos BURINI***
}

RESUMO - A doença hepática, alcoolismo e desnutrição são condições comumente associadas que interferem no metabolismo de micronutrientes. Como resultado da doença hepática pode ocorrer menor estocagem e conversão de vitaminas nas suas formas ativas, e má digestão elou má absorção. Há ainda o agravante do álcool diminuindo a ingestão e absorção de micronutrientes em virtude da redução da ingestão dietética e de sua associação com doença do intestino delgado ou pancreática. Outras causas de deficiências seriam: tratamento com drogas, peroxidação lipídica, déficit protéico, maior excreção urinária e aumento da necessidade e degradação de nutrientes. Como conseqüencias dessas deficiências, esses pacientes apresentam usualmente anemia, esteatose hepática, estresse oxidativo e imunossupressão.

DESCRITORES - Hepatopatias. Alcoolismo. Micronutrientes.

\section{INTRODUÇÃO}

Na doença hepática o comprometimento nutricional está relacionado com alterações no estado de micronutrientes, presença ou não de ingestão alcoólica e com o grau de comprometimento da função hepatocelular. Reconhecidamente, o estado vitamínico-mineral está deficiente com a redução da ingestão energética ${ }^{(41)}$, uma das principais causas de desnutrição protéico-energética na cirrose hepática ${ }^{(2,32)}$. Há ainda de se ressaltarem os hábitos alimentares distorcidos (má qualidade da dieta) dentre os alcoolistas crônicos e indivíduos com hepatopatia. Paralelamente, pode existir o déficit de micronutrientes, independente da redução da ingestão energética, pela simples adição de álcool à ingestão energética usual $^{(32)}$, de modo independente da presença de doença hepática ${ }^{(11)}$. Assim, o álcool pode ser causa tanto de desnutrição primária, pelo fato de deslocar os nutrientes da dieta, como de desnutrição secundária, por ser responsável pela má absorção e agressão celular decorrentes de sua citotoxicidade $\operatorname{direta}^{(20)}$. Pretende-se, pois, descrever as alterações do metabolismo vitamínico-mineral e suas conseqüências sobre o estado nutricional de alcoolistas sem ou com doença hepática.

Centro de Metabolismo e Nutrição (CeMeNutri) da Faculdade de Medicina da Universidade Estadual Paulista - UNESP, Botucatu, SP.

* Nutricionista. Mestre em Metabolismo e Nutrição pela UNESP.

** Professora Assistente Doutora do Departamento de Clínica Médica do Centro de Ciências da Saúde da Universidade Estadual de Londrina, PR

*** Professor Titular do Departamento de Clínica Médica da Faculdade de Medicina da UNESP. Coordenador do CeMeNutri.

Endereço para correspondência: Dr. Roberto Carlos Burini - Centro de Metabolismo e Nutrição - Faculdade de Medicina de Botucatu - UNESP - Caixa Postal 584 - 18618-970 - Botucatu, SP. 


\section{Alterações no metabolismo vitamínico-mineral}

As deficiências de micronutrientes ocorrem, com freqüência, em pacientes com doença hepática alcoólica ${ }^{(33)}$, ou em alcoolistas sem evidências de doença hepática, estando, neste caso, relacionadas ao consumo do álcool ${ }^{(11)}$. Como conseqüência destas deficiências, esses pacientes apresentam, usualmente, anemia ${ }^{(30)}$, esteatose hepática ${ }^{(25)}$, estresse oxidativo( ${ }^{(8)}$ e imunossupressão(1).

\section{Anemia}

Dentre as causas da anemia desses pacientes estão as deficiências de fatores hematopoéticos como folato, vitamina B12 e ferro $(\mathrm{Fe})$. A anemia por deficiência de Fe parece ser o sinal clínico mais característico de pacientes cirróticos com gastropatia congestiva, devido ao sangramento, crônico e periódico, da mucosa gástrica ${ }^{(5)}$. Lesões na mucosa gástrica foram detectadas em $60 \%$ dos pacientes cirróticos $^{(5)}$, sendo o sangramento agudo de varizes esofagianas a grande complicação desses pacientes ${ }^{(36)}$. Pacientes com insuficiência hepática crônica apresentam níveis diminuídos das proteínas participantes da coagulação sangüínea (fatores de coagulação: II, VII, IX e X) ${ }^{(24)}$; também o fibrinogênio e o fator V estão diminuídos na doença hepática grave ${ }^{(24)}$. As hemorragias, a possível menor ingestão de $\mathrm{Fe}$ (pela anorexia) e a provável menor secreção de eritropoietina $^{(26)}$ seriam os causadores da anemia ferropriva no paciente cirrótico ${ }^{(22)}$. Entretanto, MEANS et al. ${ }^{(23)}$, estudando pacientes com doença hepática crônica, encontraram resposta eritropoiética sérica preservada, diferente do normalmente observado na anemia da doença crônica. Esta última é mediada pelas citocinas moduladoras da imunocompetência, em especial o fator de necrose tumoral (FNT) e interferon gama (IFN $\gamma$ ) e está associada à resposta enfraquecida da eritropoietina sérica à anemia. Acrescentando-se ainda a esses fatores, a anemia do paciente cirrótico poderia ocorrer pelo hiperesplenismo e hemodiluição ${ }^{(30)}$. O agravante do álcool seria pela supressão da medula óssea na produção de células sangüíneas ${ }^{(34)}$.

A concentração reduzida de folato foi observada nos eritrócitos de $60-65 \%$ dos alcoolistas crônicos, enquanto a redução nos níveis séricos ocorreu apenas em $15 \%$ dos $\operatorname{casos}^{(4,11)}$. A deficiência nutricional de folato nesses pacientes, pode ser atribuída a menor ingestão (pela anorexia), a menor absorção intestinal (pelo etanol e desnutrição), a menor captação e retenção hepática (pela fibrose parenquimatosa) e a maior excreção urinária ${ }^{(4,29,40)}$. Os radicais livres também podem ser responsabilizados pela clivagem da molécula vitamínica $^{(27)}$ e a insuficiência hepática, pela menor estocagem e menor conversão do ácido fólico na sua forma ativa (tetrahidrofolato) $^{(6)}$. A deficiência de vitamina B12 ocorre, com menor freqüência, nos alcoolistas, devido provavelmente aos grandes estoques hepáticos ${ }^{(14)}$. A maioria dos pacientes apresenta níveis séricos normais dessa vitamina ${ }^{(11)}$ podendo haver, entretanto, pacientes com concentrações baixas no soro e no fígado ${ }^{(15)}$. O déficit tecidual dessa vitamina pode cursar com níveis circulantes subnormais, normais ou elevados ${ }^{(15)}$, possivelmente pela ação do álcool, diminuindo a retenção das cobalaminas pelos tecidos periféricos e, resultando no seu acúmulo no plasma ${ }^{(15)}$. As causas enumeradas para a deficiência dessa vitamina são: a) a ação do etanol, diminuindo a ingestão e também a absorção, e b) o aumento das suas necessidades $^{(28)}$.

\section{Estresse oxidativo, esteatose hepática e aterogênese}

Níveis circulantes reduzidos de vitamina B6 (piridoxal-5-fosfato) também constituem achado comum nesses pacientes ${ }^{(11)}$. Além de participar do metabolismo do triptofano na formação de niacina, a vitamina B6 atua, juntamente com o folato e a vitamina B12, na metabolização da homocisteína. A utilização da homocisteína ocorre via remetilação à metionina com participação de folato e vitamina B12 (metionina sintetase) ou via transulfuração à cisteína e taurina com participação da vitamina B6 (cistationina sintetase) ${ }^{(4)}$ (Figura 1). Todas essas vitaminas podem estar diminuídas no alcoolismo crônico $^{(4)}$. O acúmulo de homocisteína é o principal indicador isolado de aterogênese $\mathrm{e}^{(21)}$. A falta de metionina e dos demais fatores lipotrópicos (folato, B12, colina) pode ser responsabilizada pela esteatose hepática ${ }^{(25)}$. Além disso, na deficiência de metionina, há menor formação de S-adenosilmetionina (transmetilação) com menor ativação de folato, do tRNA, síntese de creatina, carnitina, lecitina e norepinefrina $^{(3)}$. A queda da transulfuração e formação da cisteína

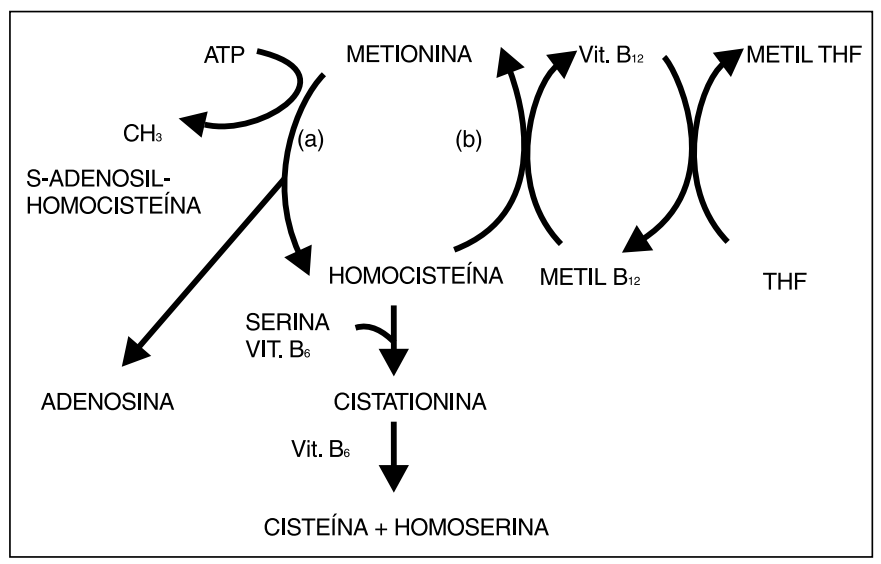

FIGURA 1 - Transmetilação (a) e remetilação(b) da metionina.

reduz os níveis de glutation, diminuindo, assim, parte importante da defesa antioxidante intracelular ${ }^{(37)}$, o que pode ser agravado pela deficiência de outros minerais como selênio, cofator da glutation peroxidase $^{(9)}$, zinco, cofator da superóxido dismutase ${ }^{(43)}$ e ferro, cofator da catalase ${ }^{(42)}$, todos deficientes em alcoolistas crônicos ${ }^{(8)}$. 
Deficiência de vitamina E, comum em pacientes alcoolistas ${ }^{(16)}$, particularmente com pancreatite crônica ${ }^{(37)}$, predispõe ainda mais o hepatócito à peroxidação lipídica ${ }^{(8)}$. $\mathrm{O}$ efeito do álcool sobre os níveis dessas vitaminas antioxidantes (beta caroteno e vitaminas $\mathrm{C}$ e E) pode depender ou não do estado nutricional do paciente ${ }^{(16,35)}$. Nos alcoolistas, além da má absorção intestinal ${ }^{(37)}$, os níveis de alfatocoferol são influenciados pela desnutrição protéico-energética ${ }^{(35)}$, menor estocagem hepática ${ }^{(38)}$ e degradação aumentada pelo hipermetabolismo hepático ${ }^{(13)}$ ou pela peroxidação lipídica ${ }^{(8)}$. Portanto, no geral, o álcool favorece o estresse oxidativo com peroxidação dos lipídios da membrana dos eritrócitos (provocando anemia) e dos hepatócitos, perpetuando a doença hepática.

\section{Hipovitaminose A e pelagra}

As deficiências de vitaminas lipossolúveis são freqüentes nos pacientes com má absorção de gorduras, alcoolismo crônico e naqueles em tratamento com colestiramina, glicocorticóides ou neomicina $^{(28)}$.

Níveis plasmáticos reduzidos de vitamina $\mathrm{A}$ e dificuldade na adaptação visual ao escuro, na cirrose hepática, podem ser atribuídos a menor mobilização hepática dessa vitamina por falta da sua proteína transportadora, o complexo proteína ligadora do retinoltranstiretina (RBP-TTR), em consequência da desnutrição protéicoenergética ou da deficiência de zinco $(\mathrm{Zn})^{(1)}$.

Verifica-se depleção das reservas hepáticas de beta caroteno e retinol em todos os estágios da hepatite de etiologia alcoólica, mesmo na ausência de alterações nos seus níveis plasmáticos ${ }^{(17,18)}$. Com a suplementação de beta caroteno há interação com o álcool resultando em hepatoxicidade caracterizada por fibrose, carcinogênese e, provavelmente, embriotoxicidade ${ }^{(19)}$.

Os níveis circulantes de $\mathrm{Zn}$ no soro dos pacientes cirróticos encontram-se usualmente reduzidos pela menor ingestão e absorção ou, mesmo, maior excreção urinária ${ }^{(31)}$. Essa deficiência agrava a deficiência de niacina pela menor síntese de ácido nicotínico, via triptofano. Saliente-se que a utilização de nicotinamida está aumentada pela metabolização hepática do álcool a acetato ${ }^{(7)}$. Juntamente com a pelagra, a neuropatia alcoólica (pela deficiência de B1) é outro sinal de hipovitaminose comum nos pacientes hepatopatas alcoolistas ${ }^{(10)}$.

\section{Imunossupressão}

Vários micronutrientes têm papel crucial na preservação da imunocompetência. As alterações imunológicas, em pacientes com doença hepática de etiologia alcoólica, podem resultar da ação direta do álcool sobre os mecanismos de barreira no trato gastrointestinal ${ }^{(39)}$, cuja principal função é controlar a flora intestinal e prevenir a entrada de antígenos no sistema $^{(39)}$. Assim sendo, os alcoolistas crônicos possuem diminuição da secreção de ácido gástrico ${ }^{(39)}$, um importante regulador do crescimento da flora intestinal, o que contribui para a incidência de supercrescimento bacteriano jejunal encontrado nesses pacientes ${ }^{(12)}$. Verificou-se, também, permeabilidade intestinal elevada a bactérias e/ou suas toxinas $^{(12)}$. A queda da defesa imunológica pode ser conseqüente, também, da desnutrição induzida pelo álcool (anorexia, diminuição da absorção, utilização, estoque e excreção de nutrientes), juntamente com os efeitos imunotóxicos diretos do álcool ${ }^{(39)}$. Dessa forma, o álcool leva à quebra da barreira intestinal, com conseqüente migração de bactérias por três mecanismos: aumento do número de bactérias no intestino, aumento de permeabilidade da mucosa intestinal e diminuição da defesa imunológica (Figura 2).

A função intestinal é complementada pelas células de Kupfer, macrófagos residentes no fígado, que têm papel central na função fagocítica durante a sepsis. Nessa condição, o álcool atua e causa dano e saturação fagocítica das células de Kupfer, resultando em estímulo antigênico produzido pela maior passagem de toxinas e bactérias para a circulação sistêmica. Em resposta, ocorre o aumento da interleucina 1 (IL-1) e FNT pelos macrófagos e monócitos, responsáveis pela ativação crônica dos linfócitos T. Como resposta há, por um lado, aumento dos níveis das imunoglobulinas séricas ( $\operatorname{IgG}$ e IgA) que possuem a capacidade de neutralização de toxinas e lise bacteriana e, por outro, evolução para a exaustão desses linfócitos, após certo tempo, causando deficiência das citocinas que estimulam as funções imune celulares, como a interleucina 2 (IL-2) e IFN $\gamma^{(1)}$. Devido a essas alterações, os pacientes com cirrose de etiologia alcoólica, evoluem com quadro de imunossupressão sistêmica $^{(1)}$ (Figura 2).

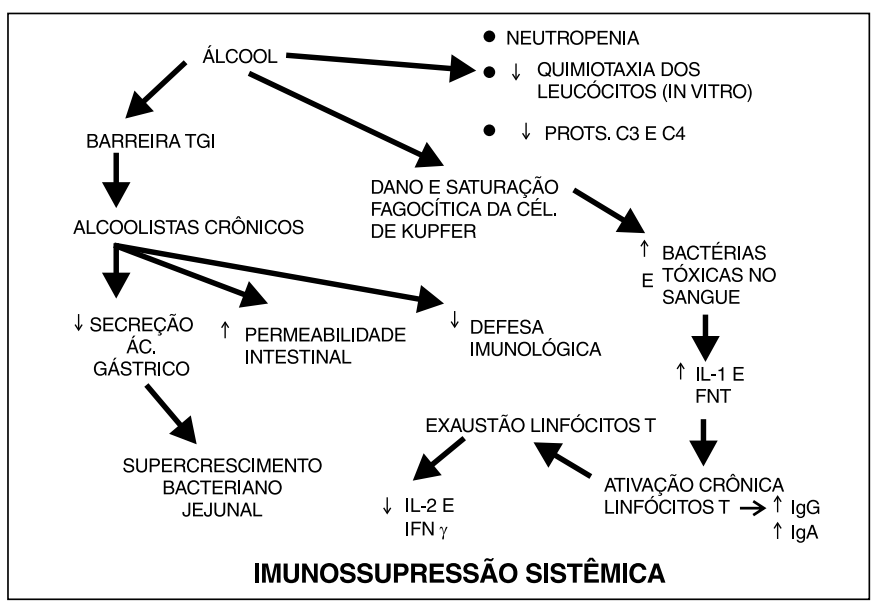

FIGURA 2 - Alterações imunológicas da doença hepática de etiologia alcoólica*.

* adaptado de Watzl e Watson ${ }^{(39)}$ e Calamita e Burini ${ }^{(1)}$ 


\section{CONSIDERAÇÕES FINAIS}

A deficiência de micronutrientes, dentre os hepatopatas crônicos, pode ser atribuída à anorexia, má absorção e às ações metabólicas do álcool. A presença do álcool agrava a anorexia e má absorção, estimula o hipermetabolismo, o estresse oxidativo e a maior excreção urinária de micronutrientes hidrossolúveis. Como conseqüências têm-se anemia, esteatose hepática, pelagra, aterosclerose e imunossupressão.

Maio R, Dichi JB, Burini RC. The impact of alcohol and chronic liver disease of micronutrients metabolism. Arq Gastroenterol 2000;37(2):120-124.

ABSTRACT - Liver disease, alcohol and malnutrition are combinations usually associated with micronutrient impairment. Chronic liver disease courses with lower storage and activation of vitamin-coenzymes related to their malabsorption. Alcohol worsens the picture by reducing food intake, increasing micronutrients utilization and decreasing their absorption secondary to either intestinal or pancreatic injuries. Other concurrent causes would be drug treatments, urinary losses, protein deficiency and oxidative stress. As consequences the clinical signs are anemia, liver steatosis, oxidative stress and immunosuppression.

HEADINGS - Liver diseases. Alcoholism. Micronutrients.

\section{REFERÊNCIAS BIBLIOGRÁFICAS}

1. Calamita Z, Burini RC. Alterações imunitárias na cirrose hepática alcoólica. Arq Gastroenterol 1995;32:79.

2. Campillo B, Bories PN, Leluan M, Pornin B, Devanlay M, Fouet P. Short-term changes in energy metabolism after 1 month of a regular oral diet in severely malnourished cirrhotic patients. Metabolism 1995;44:765.

3. Combs GF Jr. The vitamins: fundamental aspects in nutrition and health. San Diego: Academic Press; 1992. p.295, 366, 399, 404

4. Cravo ML, Glória LM, Selhub J, Nadeau MR, Camilo ME, Resende MP, Cardoso JN, Leitão CN, Mira FC. Hyperhomocysteinemia in chronic alcoholism: correlation with folate, vitamin B-12, and vitamin B-6 status. Am J Clin Nutr 1996;63:220

5. D'Amico G, Montalbano L, Traina M, Pisa R, Menozzi M, Spanò C, Pagliaro L. Natural history of congestive gastropathy in cirrhosis. The Liver Study Group of V. Cervello Hospital. Gastroenterology 1990;99:1558.

6. Dichi I, Papini SJ, Dichi JB, Resende TA, Burini RC. Estado nutricional de pacientes portadores de cirrose hepática. Relação entre a gravidade da doença com a piora nutricional avaliada por indicadores antropométricos e bioquímicos. Rev Bras Nutr Clin 1991;6:29.

7. Dichi JB, Burini RC. Metabolismo do etanol e estado energético de pacientes cirróticos. Rev Metab Nutr 1995;2:69.

8. Dichi JB, Burini RC. Alterações da defesa antioxidante na hepatopatia alcoólica. Rev Metab Nutr 1996;3:50.

9. Dworkin B, Rosenthal WS, Jankowsky RH, Gordon GG, Haldea D. Low blood selenium levels in alcoholics with and without advanced liver disease. Correlations with clinical and nutritional status. Dig Dis Sci 1985;30:838.

10. Feinman L, Lieber CS. Nutrition and diet in alcoholism. In: Shils ME, Olson JA, Shike M. Modern nutrition in health and disease. 8.ed. Philadelphia: Lea \& Febiger; 1994. v.2, p.1081.

11. Glória L, Cravo M, Camilo ME, Resende M, Cardoso JN, Oliveira AG, Leitão $\mathrm{CN}$, Mira FC. Nutritional deficiencies in chronic alcoholics: relation to dietary intake and alcohol consumption. Am J Gastroenterol 1997;92:485.

12. Gomez F, Ruiz P, Schreiber AD. Impaired function of macrophage Fcg receptors and bacterial infection in alcoholic cirrhosis. N Engl J Med 1994;331:1122.
13. Hagen FB, Bjorneboe A, Bjorneboe GE, Drevon CA. Effect of chronic alcohol consumption on the content of alpha-tocopherol in subcellular fractions of rat liver. Alcohol Clin Exp Res 1989;13:246.

14. Halsted CH, Keen CL. Alcoholism and micronutrient metabolism and deficiences. Eur J Gastroenterol Hepatol 1990;2:399.

15. Kanazawa S, Herbert V. Total corrinoid, cobalamin (vitamin B12), and cobalamin analogue levels may be normal in serum despite cobalamin depletion in liver in patients with alcoholism. Lab Invest 1985;53:108.

16. Lecomte E, Grolier P, Herberth B, Pirollet P, Musse N, Paille F, Braesco V, Siest $\mathrm{G}$, Artur Y. The relation of alcohol consumption to serum carotenoid and retinol levels. Effects of withdrawal. Int Vitamim Nutr Res 1994;64:170.

17. Leo MA, Lieber CS. Hepatic vitamin A depletion in alcoholic liver injury. N Engl J Med 1982;307:597.

18. Leo MA, Rosman AS, Lieber CS. Differential depletion of carotenoids and tocopherol in liver disease. Hepatology 1993;17:977.

19. Leo MA, Lieber CS. Alcohol, vitamin A, and b-carotene: adverse interactions, including hepatotoxicity and carcinogenicity. Am J Clin Nutr 1999;69:1071.

20. Lieber CS. Alcohol and the liver: 1994 update. Gastroenterology 1994;106:1085.

21. Masser PA, Taylor LM, Ponter JM. Importance of elevated plasma homocysteine levels as a risk factor for atherosclerosis. Ann Thorac Surg 1994;58:1240.

22. Means RT, Krantz SB. Progress in understanding the pathogenesis of the anemia of chronic disease. Blood 1992;80:1639.

23. Means RT, Mendenhall CL, Worden BD, Moritz TE, Chedid A. Erythropoietin and cytokine levels in the anemia of severe alcoholic liver disease. Alcohol Clin Exp Res 1996;20:355.

24. Mezey E. Liver disease and nutrition. Gastroenterology 1978;74:770.

25. Mezey E. Interaction between alcohol and nutrition in the pathogenesis of alcoholic liver disease. Semin Liver Dis 1991;11:340.

26. Miller CB, Jones RJ, Piantadosi S, Abeloff MD, Spivak JL. Decreased erythropoietin response in patients with the anemia of cancer. N Engl J Med 1990;322:1689.

27. Mira ML, Manso CF. Álcool e radicais livres de oxigénio. Acta Med Port 1993;6:193.

28. Müller M. Malnutrition in cirrhosis. J Hepatol 1995;23 Suppl 1:31.

29. Nielsen K, Kondrup J, Martinsen L, Stilling B, Wikman B. Nutritional assessment and adequacy of dietary intake in hospitalized patients with alcoholic liver cirrhosis. Br J Nutr 1993;69:665. 
30. Petz LD. Hematologic aspects of liver disease. Curr Opin Gastroenterol 1989;5:372.

31. Rodrigues-Moreno F, Gonzalez-Reimers E, Santolaria-Fernandez F, GalindoMartin L, Hernandez-Torres, Batista-Lopez N, Molina-Perez M. Zinc, copper, manganese, and iron in chronic alcoholic liver disease. Alcohol 1997;14:39.

32. Sarin SK, Dhingra N, Bansal A, Malhotra S, Guptan RC. Dietary and nutritional abnormalities in alcoholic liver disease: a comparison with chronic alcoholics without liver disease. Am J Gastroenterol 1997;92:777.

33. Shenker S, Halff GA. Nutritional therapy in alcoholic liver disease. Sem Liver Dis 1993;13:196.

34. Sullivan LW, Herbert V. Suppression of hematopoesis by ethanol. J Clin Invest 1964;43:2048

35. Tanner AR, Bantok I, Hinks L, Lloyd B, Turner NR, Wright R. Depressed selenium and vitamin E levels in a alcoholic population. Dig Dis Sci 1986;31:1307.

36. Thomsen BL, Moller S, Sorensen TIA. Optimized analysis of recurrent bleeding and death in patients with cirrhosis and esophageal varices. Copenhagen esophageal varices sclerotherapy project. J Hepatol 1994;21:367.

37. Van Gossum A, Closset P, Noel E, Cremer M, Neve J. Deficiency in antioxidant factors in patients with alcohol-related chronic pancreatitis. Dig Dis Sci $1996 ; 41: 1225$
38. Ward RJ, Jutla J, Peters TJ. Antioxidants status in alcoholic liver disease. Adv Biosci 1989;76:343.

39. Watzl B, Watson RR. Role of alcohol abuse in nutritional immunosuppression. J Nutr 1992;122 Suppl 3:733.

40. Weir DG, McGing PG, Scott JM. Folate metabolism, the enterohepatic circulation and alcohol. Biochem Pharmacol 1985;34:1.

41. Whitehead RG. Lowered energy intake and dietary macronutrient balance:potencia consequences for micronutrient status. Nutr Rev 1995;53:S2.

42. Yu BP. Cellular defenses against damage from reactive oxygen species. Phisiol Rev 1994;74:139.

43. Zimmermann A, Zhao M, Matter K, Laissue JA. Differential expression of $\mathrm{Cu} / \mathrm{Zn}$ and $\mathrm{Mn}$ superoxide dismutase in alcoholic liver disease in man: potencial protective role of Mn superoxide dismutase. J Hepatol 1994;21 Suppl 1:S80.
Recebido para publicação em 23/2/1999. Aprovado para publicação em 21/3/2000. 